\title{
COMMENT
}

\section{The 2020 Census and the child undercount: a threat to pediatric research and the health and wellbeing of children}

\author{
Judy L. Aschner ${ }^{1}$, Jean L. Raphael ${ }^{2}$ and Shale L. Wong ${ }^{3}$ on behalf of the Pediatric Policy Council \\ Pediatric Research (2019) 86:289-290; https://doi.org/10.1038/s41390-019-0477-6
}

As pediatricians and pediatric investigators, one of our responsibilities is to advocate for children who often have no voice and no political clout. Many of us are engaged in advocacy efforts to combat hazards that place current and future generations of children at risk. From climate change, to poverty, to gun violence, to funding shortfalls for pediatric research, there seems to be no shortage of critical issues that pose a threat to child health and would benefit from coordinated advocacy efforts on the part of pediatricians and the pediatric research community. ${ }^{1-3}$ One such time-sensitive and urgent threat in need of coordinated action is the possibility of a large child undercount in the looming 2020 Census. The concern about undercount is further compounded by the Trump administration's proposal to add a question about U.S. citizenship to the upcoming Census.

Article 1, Section 2 of the U.S. Constitution mandates a decennial census to count all individuals living in the United States. Prior U.S. Census data show that children, particularly those aged $<5$ years, are at the greatest risk of not being counted. According to the U.S. Census Bureau, the undercount of young children has gotten worse between the 1980 Census and the 2010 Census. In 1980, $1.4 \%$ of children aged $<5$ years were not counted compared with nearly $5 \%$ in 2010 . More than 2 million children (10\%) were omitted from the 2010 Census while close to 1 million children, such as those living in joint custody situations and those living on college campuses, were counted twice. ${ }^{4}$ Among young children, aged $0-4$ years, the net child undercount was about 1 million-the highest undercount of any age group.

The implications of a significant child undercount are far reaching. Federal and state agencies use Census data to populate formulas involving population and poverty, which in turn, are used to allocate funding. Thus the failure to count all children imperils numerous health, educational, and community programs. The Census data also determines congressional representation for the next 10 years and is used for state and local redistricting. Inaccurate Census data undermines critical areas of pediatric research. Census data are used by investigators for epidemiologic, health services, environmental, and population-based research. Children's Hospitals and health-care systems use census data for quality improvement initiatives, needs assessments, staffing decisions, and public health research. The data from the 2020 Census will direct between $\$ 675$ billion and $\$ 800$ billion annually in Federal funding for programs that protect our most vulnerable children. The George Washington Institute of Public Policy has published a report showing that, depending on the State, annual lost Medicaid funds range from
$\$ 533$ to $\$ 2,309$ for each person uncounted by the census. ${ }^{5}$ Since 37 million children in the U.S. are covered by Medicaid, ${ }^{6}$ a large child undercount would affect the ability of many States to meet the health-care needs of its children. Table 1 lists some of the federally sponsored programs that rely on the data from the Census to distribute funding that serve children. Groups who are not counted will not get their fair share of resources or representation.

The reasons why children are not counted are numerous and complex. At the highest risk of not being counted are newborn infants, children who split time between two homes, children who live or stay with another family or with another relative such as a grandparent, children who live in a lower-income household or in a household with young parents or a young, single mother, and children who live in a non-English or limited-English speaking household. Interestingly, many households who complete the Census questionnaire for the adults in their family do not include their young children.

As pediatric researchers, providers, and child advocates, what can we do to minimize the child undercount and the concomitant risk that children and vulnerable populations will not get their fair share of resources? As trusted voices in our communities, we can convey to parents the importance of completing the census survey and including all family members, including young children. We can explain to families that, when individuals are missed, communities do not receive their fair share of resources or representation. We can emphasize that parents and caregivers should include babies on census forms, even if they are still in the hospital on April 1, 2020. This is an educational and advocacy opportunity that the pediatric workforce can help to address. The Census data are protected by Title 13, which means, by law, respondent information is strictly confidential. ${ }^{7}$ Pediatric providers can reassure families that the Census Bureau does not share information with immigration enforcement agencies and law enforcement agencies like the police or the $\mathrm{FBI}$ nor allow this information to be used to determine eligibility for government benefits.

The Federation of Pediatric Organizations (FOPO) is an umbrella organization representing the collective voices of the Society for Pediatric Research (SPR), the American Pediatric Society (APS), the American Board of Pediatrics (ABP), the American Academy of Pediatrics (AAP), the Academic Pediatric Association (APA), the Association of Medical School Pediatric Department Chairs (AMSPDC), and the Association of Pediatric Program Directors (APPD). FOPO has partnered with the U.S. Census Bureau to inform and engage the pediatric community about the undercount

\footnotetext{
'Department of Pediatrics, Joseph M Sanzari Children's Hospital at Hackensack University Medical Center, Hackensack Meridian Health School of Medicine, Hackensack, NJ 07601, USA; ${ }^{2}$ Center for Child Health Policy and Advocacy, Baylor College of Medicine, Houston, TX, USA and ${ }^{3}$ Department of Pediatrics, University of Colorado School of Medicine, Aurora, CO, USA

Correspondence: Judy L. Aschner (judy.aschner@hackensackmeridian.org)
}

Received: 4 June 2019 Accepted: 14 June 2019

Published online: 22 June 2019 
Table 1. Some programs that use the census data to determine funding distributions

Medicaid

Children's Health Insurance Program (CHIP)

Supplemental Nutrition Assistance Program (SNAP)

Title I funding to schools in low-income communities

Temporary assistance to needy families

IDEA special education funding for children with disabilities

Head start

Foster care

Adoption assistance

National school lunch and school breakfast programs

Child care programs

Maternal and Child Health Services Block Grants to the States

Special Education Grants for infants and families

States, localities, and businesses use census data to plan, including where to put new schools or to open new stores

For a more complete list of programs that received federal assistance distributed using Census Bureau Data in 2015-2016, see https://www2.census.gov/ programs-surveys/decennial/2020/program-management/working-papers/Uses-of-Census-Bureau-Data-in-Federal-Funds-Distribution.pdf

Table 2. Websites with information about the 2020 Census and the child undercount

https://countallkids.org/

https://www.commerce.gov/news/blog/2018/10/us-census-bureau-recruits-key-partner-improve-2020-count-children

https://www2.census.gov/programs-surveys/decennial/2020/program-management/working-papers/Uses-of-Census-Bureau-Data-in-Federal-Funds-Distribution.pdf

http://www.fopo.org/

https://www.oyez.org/cases/2018/18-966

https://www.childrenshospitals.org/Newsroom/Childrens-Hospitals-Today/Spring-2019/Articles/2020-Census-Data-Impact-Childrens-Health-Care?

utm_source=exm\&utm_medium =email\&utm_campaign $=$ cht\&utm_content $=051419$

https://gwipp.gwu.edu/counting-dollars-2020-role-decennial-census-geographic-distribution-federal-funds

https://www.childrenshospitals.org/Newsroom/Childrens-Hospitals-Today/Articles/2019/04/Census-101-The-2020-Census-and-Why-it-Matters?

utm_source $=$ exm\&utm_medium $=$ email\&utm_campaign $=$ cht\&utm_content $=050219$

of young children, its consequences, and what we as pediatricians and pediatric researchers can do to help address it and assure that all kids are counted in the 2020 Census. After the Supreme Court rules on whether or not to add a citizenship question to the Census survey, the FOPO website (http://www.fopo.org/) will serve as a communications platform and hub with information, flyers, talking points, and tool kits that pediatricians, trainees, and researchers can share with families on the benefits of completing the census survey. This will include materials that can be shared in a pediatrician's office, subspecialty ambulatory clinic, hospital, or research venue to remind parents/family/caregivers to count their young children. FOPO will develop public service announcements on the importance of counting young children in the 2020 Census. Prominent pediatricians across the country are encouraged to create Public Service Announcements for their local communities on the importance of counting children and a call to action to pediatricians on what they can do in their own offices to help address the challenge. The academic pediatric community is encouraged to sponsor presentations, workshops, and exhibit booths at our national meetings. FOPO will disseminate information via social media channels, press kits, tweets, ads, and commentaries, such as this one, in our journals.

Table 2 lists publicly available websites where more can be learned about the challenges and implications of the child undercount, the resources for addressing it, and how to advocate around an issue that is critical to research initiatives and the future of our children. All kids count. We need to do our part to assure that all kids are counted in the 2020 Census.

\section{ADDITIONAL INFORMATION}

Competing interests: The authors declare no competing interests.

Publisher's note: Springer Nature remains neutral with regard to jurisdictional claims in published maps and institutional affiliations.

\section{REFERENCES}

1. Flores, G. \& Lesley, B. Children and U.S. federal policy on health and health care: seen but not heard. JAMA Pediatr. 168, 1155-1163 (2014).

2. Jones, N. L. et al. Life course approaches to the causes of health disparities. Am. J. Public Health 109(S1), S48-S55 (2019).

3. Linton, J. M., Ameenuddin, N. \& Falusi, O. Pediatricians awakened: addressing family immigration status as a critical and intersectional social determinant of health. Am. J. Bioeth. 19, 69-72 (2019).

4. Busenbark, M. How 2020 Census data affects children's health care. Children's Hospital Association https://www.childrenshospitals.org/Newsroom/ChildrensHospitals-Today/Spring-2019/Articles/2020-Census-Data-Impact-Childrens-Health-Care? utm_source=exm\&utm_medium $=$ email\&utm_campaign $=$ cht\&utm_content $=051419$ (2019).

5. Reamer, A. Counting for dollars 2020: the role of the decennial Census in the geographic distribution of federal funds. George Washington Institute of Public Policy https://gwipp.gwu.edu/counting-dollars-2020-role-decennial-censusgeographic-distribution-federal-funds (2019).

6. Medicaid and CHIP Payment and Access Commission. CHIP enrollment in CHIP and Medicaid by states, FY2016. Macpac.gov https://www.macpac.gov/wp-content/ uploads/2015/01/EXHIBIT-32.-Child-Enrollment-in-CHIP-and-Medicaid-by-State-FY2017-thousands.pdf (2019).

7. The United States Census Burea. Privacy and confidentiality https://www.census. gov/history/www/reference/privacy_confidentiality/title_13_us_code.html (2019). 